\title{
The Effect of Ureteric Stenting on Female Sexual Function: A Prospective Cohort Study
}

\author{
Zehra Kazmi $^{1}$, Daniya Umer ${ }^{2}$, M Hammad Ather ${ }^{1}$ \\ 1. Urology, The Aga Khan University, Karachi, PAK 2. Surgery, The Aga Khan University, Karachi, PAK
}

Corresponding author: Zehra Kazmi, zehra-kazmi@hotmail.com

\begin{abstract}
\section{Background and objective}

Urolithiasis is a highly prevalent disease worldwide, with Pakistan belonging to the stone belt of Asia. The usage of the double J (DI) stent is highly effective when it comes to the management of urolithiasis. However, studies investigating the side effects of DJ stent placement on sexual function in individuals are scarce. In this study, we aimed to assess the impact of DJ stent placement on sexual function in women.
\end{abstract}

\section{Methods}

After obtaining ethical approval, a prospective study was conducted at a university hospital from June 2018 to September 2019. All sexually active women requiring semi-rigid ureteroscopy (URS) or flexible URS [retrograde intrarenal surgery (RIRS)] were enrolled. Women with DJ stent placement (Group A) were compared to women who did not require DJ stent (Group B). The outcome variable was to assess temporary sexual dysfunction after DJ stent placement using the standardized Female Sexual Functionality Index (FSFI) in English or its validated vernacular version. The FSFI was completed at four weeks, and again at three months, following URS/RIRS.

\section{Results}

Of the 106 sexually active women initially included in the study, 69 were found to be eligible for final analysis. In Group A, the mean FSFI score at the initial presentation was $31.54 \pm 4.37$. The mean FSFI score at four weeks was lower compared to the baseline score $(0$ time $): 13.96 \pm 5.5(\mathrm{p}<0.05)$. At three months, the mean FSFI score returned to near baseline at $32.053 \pm 5.35$ with no significant difference $(\mathrm{p}=0.65)$. In comparison to women in Group B, the mean FSFI score at four weeks was significantly lower in Group A (28.87 $\pm 6.59 \mathrm{vs}$. $13.96 \pm 5.49 ; \mathrm{p}<0.05)$. However, there was no significant difference between the mean FSFI scores at any of the three time points within Group B.

\section{Conclusion}

DJ stent insertion results in transient postoperative sexual dysfunction in women, which resolves spontaneously within a span of three months after stent removal.

Review began 10/09/2020 Review ended 10/15/2020 Published 10/21/2020

\section{() Copyright 2020}

Kazmi et al. This is an open access article distributed under the terms of the Creative Commons Attribution License CC-BY 4.0., which permits unrestricted use, distribution, and reproduction in any medium, provided the original author and source are credited.

Categories: Urology, Other, Epidemiology/Public Health Keywords: sexual dysfunction, fsfi, fsd, dj stent, ji stent

\section{Introduction}

Upper tract urolithiasis is a highly prevalent disease worldwide with prevalence rates ranging from 7 to $13 \%$ in North America, 5-9\% in Europe, and 1-5\% in Asia [1]. For most stones managed by endourological procedures, a double J (DJ) stent is an indispensable device. It offers effective drainage of the urinary tract and is very convenient to use. However, DJ stents are associated with frequent side effects and have a significant impact on the patient's quality of life [2]. There are many papers published on the impact of an indwelling DJ stent on the patient's quality of life [3,4]. In addition, contemporary literature also describes various effective treatment modalities to help with stent-related symptoms $[5,6]$. However, there is a dearth of literature evaluating the impact of DJ stents on sexual function in women.

The aim of our study is to prospectively analyze the impact of an indwelling DJ stent on female sexual function. In most societies, female sexual dysfunction (FSD) remains an issue that is rarely discussed. In this day and age, there is increasing awareness among women regarding sexual health, and they are now more forthcoming when it comes to such sensitive issues. The Female Sexual Function Index (FSFI) [7] has been translated into and validated in the vernacular [8]. It is a 19-segment-based, self-administered questionnaire consisting of six domains, which has been validated for use in diagnosis and categorization of FSD [9].

\section{Materials And Methods}




\section{Study design}

This prospective cohort study was conducted at the Aga Khan University Hospital in Karachi, Pakistan. The sample size was calculated using the OpenEpi program (version 3), with a power of $80 \%$ and a two-sided confidence interval of $95 \%$. The study group consisted of 106 consecutive sexually active female patients presenting to our hospital with a diagnosis of ureteric calculi and requiring ureteroscopy (URS). After obtaining approval from the Ethical Review Committee, all sexually active females presenting with ureteral stone diseases requiring URS were enrolled in the study. They were equally divided into two groups: Group A required stent placement for approximately four weeks postoperatively, while Group B consisted of women who did not require any DJ stent placement. All of these women were followed up prospectively up to a period of three months. The FSFI questionnaire (a previously validated, brief, and self-administered 19-item questionnaire assessing six domains of female sexual function) was used to evaluate FSD. It was specifically designed to assess sexual dysfunction over the preceding four weeks.

\section{Participants}

Patients who had a complicated URS \{associated with a high-grade injury to the ureter [postureteroscopic lesion scale grade 2+ (PULS 2+)], incomplete stone fragmentation, bilateral stents, Clavien-Dindo grade III + complications\}, urinary tract infection (UTI) within four weeks prior to the procedure, pregnancy, previous history of endourological procedures, pelvic surgery or radiotherapy, or those women who were lost to follow-up, were excluded from the study. The indications for stent placement were left to the discretion of the operating surgeon. These included the presence of ureteral wall edema, suspicion of stone migration, impacted stones, or patients from outside the city who required traveling for further follow-up and management.

\section{Data collection}

All patients were requested to fill in the FSFI form at the initial visit (time 0), which was taken as their baseline score; the scores were again recorded in two subsequent instances: at four weeks and at three months after the procedure. The outcome variables were to assess the impact of stent placement on sexual function and to compare it between the two groups.

\section{Data analysis}

Statistical analysis was performed using Stata/SE 12.1 and Microsoft Excel (Microsoft Corporation, Redmond, WA). Data were stratified quantitatively and descriptively. Quantitative parameters included the FSFI total scores and domain scores for the stent and no-stent groups. These were compared using the Student's paired t-test, Wilcoxon Rank Sum Test, and chi-squared test as applicable. Descriptive parameters included age, site of the stent, duration of stenting (in days), and the language of the FSFI questionnaire. A p-value of less than 0.05 was considered to be statistically significant.

\section{Results}

A total of 106 sexually active women were initially included in the study. Out of this, 37 women were excluded; the most common reason was the refusal to participate because of hesitation to discuss intimate details. The final study population included 69 women (36 in group A and 33 in group B). Patients were given the option to fill in the FSFI form either in the vernacular or English, based on their preference. Overall, the English form was filled in by $55 \%$ of the patient cohort. The average age of women in group A was $42.83 \pm 10.7$ years and that in group B was $45.21 \pm 12.68$ years, and no significant difference was found between the two groups in terms of age $(\mathrm{p}=0.4)$. The demographic data of the participants are shown in Table 1. Semi-rigid URS was performed in $47.8 \%$ of women in Group A and $33.3 \%$ in Group B, whereas retrograde intrarenal surgery (RIRS) was performed in $4.3 \%$ of women in Group A and $14.5 \%$ in Group B. Using the chisquared test, a significant association was found in terms of the type of procedure performed between groups, where RIRS was more frequently performed in Group B as compared to Group A ( $\mathrm{p}<0.04)$.

The average duration of the DJ stent insertion in participants from Group A was 37 days. Table 2 shows a comparison of the domains of sexual function in both groups, and each one of the six domains (desire, arousal, lubrication, orgasm, satisfaction, and pain) showed that there was a significant association between sexual dysfunction and the presence of indwelling DJ stent $(\mathrm{p}<0.05)$.

After three months of the procedure, the FSFI score returned to near baseline (0 time) in Group A. In Group A, the mean FSFI score at the initial presentation was $31.54 \pm 4.37$. The mean FSFI score at four weeks was significantly lower compared to the baseline score at 0 time: $13.96 \pm 5.5(\mathrm{p}<0.05)$. At three months, the FSFI score returned to baseline at $32.053 \pm 5.35$ with no significant difference $(\mathrm{p}=0.65)$. In comparison to women in Group B, the mean FSFI score at four weeks was significantly lower in Group A (28.87 \pm 6.59 vs. 13.96 $\pm 5.49 ; \mathrm{p}<0.05)$. However, there was no significant difference between the mean FSFI scores at any of the three time points within Group B (Figure 1). 


\section{Cureus}

\begin{tabular}{|c|c|c|c|}
\hline Parameters & Group A & Group B & P-value \\
\hline Age in years, average $\mathrm{SSD}$ & $42.83 \pm 10.7$ & $45.21+12.685$ & 0.402 \\
\hline Location of stone: proximal, mid, distal; n (\%) & $17(24.6 \%), 8(11.6 \%), 11(15.9 \%)$ & $14(20.3 \%), 3(4.4 \%), 16(233.2 \%)$ & \\
\hline Procedure: RIRS, URS; n (\%) & $3(4.3 \%), 33(47.8 \%)$ & $10(14.5 \%), 23(33.3 \%)$ & \\
\hline Site of stent insertion: right, left; n & 21,15 & $\cdots$ & \\
\hline Stenting duration in days, average $\pm S D$ & $37.02 \pm 12.3$ & $\cdots$ & \\
\hline Language; English, vermacular; n (\%) & $19(27.5 \%), 17(24.6 \%)$ & $19(27.5 \%), 14(20.3 \%)$ & \\
\hline
\end{tabular}

\section{TABLE 1: Demographic characteristics of the patient cohort}

Group A: patients with stent; Group B: patients with no stent

RIRS: retrograde intrarenal surgery; URS: ureteroscopy; SD: standard deviation

\section{FSFI: Group A vs Group B}

Group A Group B

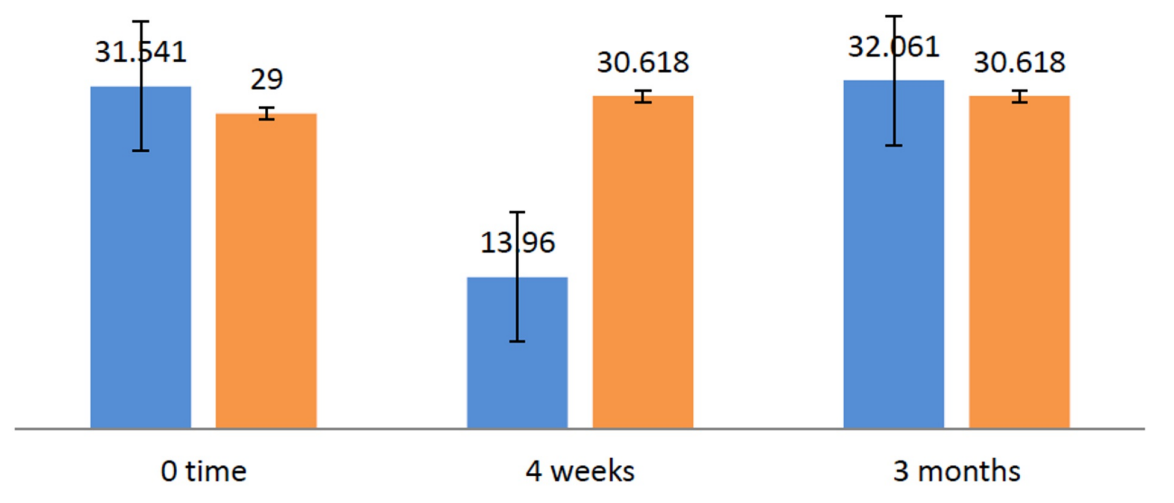

FIGURE 1: FSFI score before procedure (0 time) and after procedure (at four weeks and at three months)

FSFI: Female Sexual Functionality Index

Figure 2 compares the procedures performed with the sites of surgery. 


\section{Cureus}

\section{Surgical Procedure and Site}

25

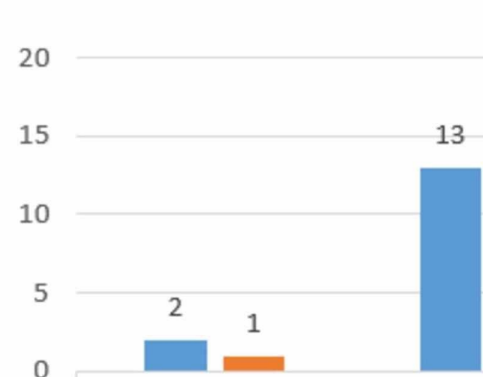

RIRS
20

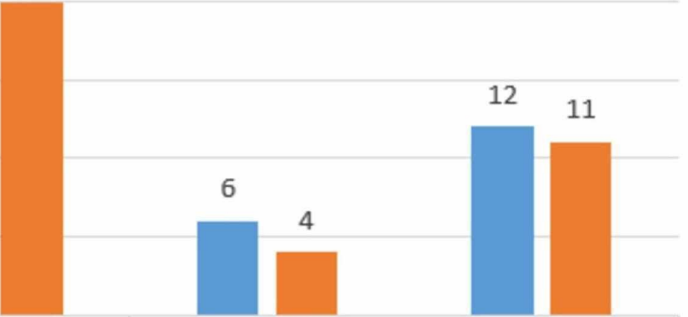

URS

RIRS

URS

Group B

Group A

- Left $\square$ Right

\section{FIGURE 2: Surgical procedures and sites}

RIRS: retrograde intrarenal surgery; URS: ureteroscopy

We further evaluated the FSFI scores in a subdomain-based manner including sexual desire (FSFI-SD), sexual arousal (FSFI-SA), sexual lubrication (FSFI-SL), sexual orgasm (FSFI-SO), general satisfaction (FSFI-SS), and pain (FSFI-SP). These scores demonstrated a statistically significant difference $(p<0.05)$ when compared between the two groups at four weeks post-procedure (Table 2). Total FSFI values and subdomains values of the females obtained before and after the stent insertion are presented in Table 3.

\begin{tabular}{|c|c|c|c|}
\hline \multicolumn{2}{|l|}{ Domains } & Postoperative score (at 4 weeks), mean \pm SD & P-value \\
\hline \multirow{2}{*}{ FSFI-SD } & Group A & $3.383 \pm 0.963$ & \multirow{2}{*}{$<0.05$} \\
\hline & Group B & $4.18 \pm 1.05$ & \\
\hline \multirow{2}{*}{ FSFI-SA } & Group A & $2.63 \pm 1.051$ & \multirow{2}{*}{$<0.05$} \\
\hline & Group B & $4.481 \pm 1.394$ & \\
\hline \multirow{2}{*}{ FSFI-SL } & Group A & $2.225 \pm 1.730$ & \multirow{2}{*}{$<0.05$} \\
\hline & Group B & $4.972 \pm 1.4792$ & \\
\hline \multirow{2}{*}{ FSFI-SO } & Group A & $1.533 \pm 1.5667$ & \multirow{2}{*}{$<0.05$} \\
\hline & Group B & $4.860 \pm 1.4735$ & \\
\hline \multirow{2}{*}{ FSFI-SS } & Group A & $2.233 \pm 1.1084$ & \multirow{2}{*}{$<0.05$} \\
\hline & Group B & $5.345 \pm 0.870$ & \\
\hline \multirow{2}{*}{ FSFI-SP } & Group A & $2.22 \pm 1.869$ & \multirow{2}{*}{$<0.05$} \\
\hline & Group B & $5.030 \pm 1.319$ & \\
\hline \multirow{2}{*}{ FSFI-t } & Group A & $13.96 \pm 5.5$ & \multirow{2}{*}{$<0.05$} \\
\hline & Group B & $30.618 \pm 7.027$ & \\
\hline
\end{tabular}

TABLE 2: Total FSFI and subdomain scores at four weeks: comparison between Group A and Group B

FSFI: Female Sexual Function Index; SD: sexual desire; SA: arousal; SL: lubrication, SO: orgasm; SS: satisfaction; SP: pain; t: total; SD: standard deviation 


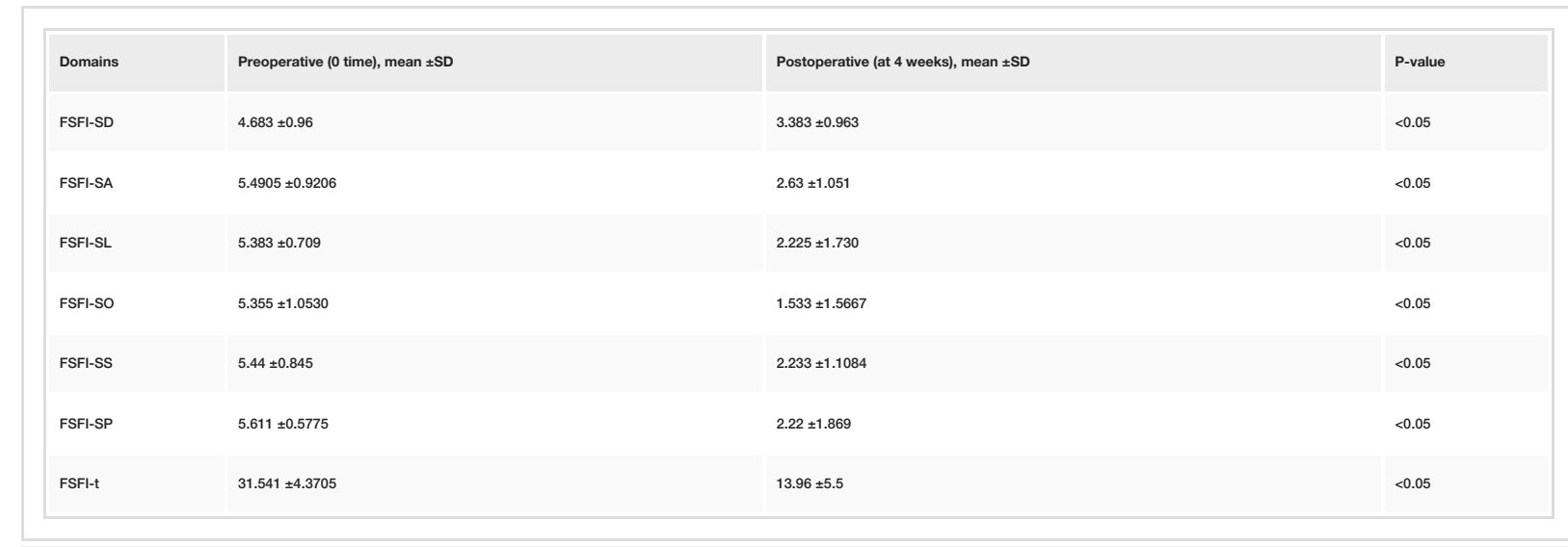

TABLE 3: Total FSFI and subdomain scores before and after DJ stent insertion (Group A)

FSFI: Female Sexual Function Index; SD: sexual desire; SA: arousal; SL: lubrication, SO: orgasm; SS: satisfaction; SP: pain; t: total; SD: standard deviation

\section{Discussion}

Interventions for ureterolithiasis depend upon many factors: location, size and stone burden, symptomatic status, and failure of conservative measures. URS is a commonly performed intervention in the management of ureteral stones [10,11]. Endourological interventions for the ureter are often accompanied by the placement of DJ stents in order to decrease complications related to stone fragmentation, to allow better drainage of the kidney, and to decrease the need for pain killers following URS [12]. DJ stent insertion may have a significant impact on the patient's quality of life [3,4]. The ureteric stent symptom questionnaire (USSQ) is a validated and reliable instrument to evaluate the impact of different types of stents commonly used in urology [13].

The so-called "stent-related symptoms" range from mild discomfort, dysuria, and hematuria, to more significant morbidities, such as UTI, reflux, mal-positioning, fracture, and encrustation [7]. In addition to all of these, a DJ stent is also known to have a significant impact on sexual function in both genders [14]. This aspect is rarely talked about, especially in certain cultures. It results in psychological disturbance, thereby deeply influencing the patient's quality of life [3].

We evaluated the effect of routine ureteric stenting on female sexual function. We used the standard, validated FSFI questionnaire in English and Urdu, to specifically document the level of dysfunction in sexually active women. All participants were given the choice to fill the form in either language. A significant decline in the total FSFI scores at four weeks after DJ stent placement was observed. It is as yet unclear as to how the DJ stent affects sexual function. One possibility is the presence of bothersome lower urinary tract symptoms resulting in secondary deterioration of sexual function [15].

The results of our study are comparable with the very few other studies conducted in other parts of the world. No similar study has been conducted to look at the effect of DJ stent insertion on women in our part of the world, and our results shall have a significant impact on patient counseling in Pakistan. Our study is also unique in that it made a comparison of FSD between two separate patient cohorts (stented versus nonstented), at three points in time.

In a cohort study conducted by Fawzi et al. in Indonesia, a significant decline in sexual function in a singlestented group of both men and women was observed [16]. Bolat et al. [17] found no correlation between URS and sexual dysfunction in their study where the International Index for Erectile Function (IIEF) for men and FSFI for women were used at one month and three months after the procedure. They reported dysfunction at one month, which showed improvement at three months, and hence concluded that the difference was most likely due to DJ stent [7]. We obtained similar results from our study, as the non-stented women (Group B) who only underwent URS reported no sexual dysfunction at any point in time. Eryildirim et al. have reported similar results in both male and female patients [11]. A notable difference in both IIEF and FSFI scores was reported before and after the installation of DJ stents. Sighinolfi et al. also obtained similar results; however, they evaluated patients before and approximately four weeks after the DJ stent insertion [3].

Our study has some limitations. The reported sample size consisted of a small group of women, and this may have induced bias. The main reason for so many participants dropping out of the study was because of the sensitive nature of this subject in our region. Secondly, any dysfunction due to the stent material and design was not evaluated, as we used only one type of stent in the stented group. More extensive studies need to be done to look at this aspect in further detail in association with the type of stents, and the effect of 
menopause or other physiologic conditions having an impact on female sexual function. Nevertheless, our preliminary analysis provides valuable insights into a subject that is usually neglected in our part of the world. Looking at the sparse amount of literature available on this "taboo" subject, we believe our results shall contribute to improved patient counseling in this part of the world.

There is a return to baseline sexual function after stent removal by three months, as indicated by our results and that of most of the reported series. Our findings indicate that women with stents were affected more when compared with the women who did not get a DJ stent; however, there was a return to baseline function three months post-procedure. This indicates that the dysfunction is temporary in nature but persistent throughout the stent-indwelling time. However, patients should be informed about this possible outcome during the general preoperative counseling.

\section{Conclusions}

Based on our findings, the use of a DJ stent after an endourological procedure causes temporary sexual dysfunction. There is a need to standardize indications for stenting following URS or RIRS, with particular emphasis on shorter operative times and minimal ureteric trauma. In cases where stenting is unavoidable, patients need to be informed in advance about this adverse effect as well.

\section{Additional Information \\ Disclosures}

Human subjects: Consent was obtained by all participants in this study. The Aga Khan University Ethical Review Committee issued approval 2018-0290-415. This project was approved by the Ethical Review Committee at the Aga Khan University. Animal subjects: All authors have confirmed that this study did not involve animal subjects or tissue. Conflicts of interest: In compliance with the ICMJE uniform disclosure form, all authors declare the following: Payment/services info: All authors have declared that no financial support was received from any organization for the submitted work. Financial relationships: All authors have declared that they have no financial relationships at present or within the previous three years with any organizations that might have an interest in the submitted work. Other relationships: All authors have declared that there are no other relationships or activities that could appear to have influenced the submitted work.

\section{References}

1. Sorokin I, Mamoulakis C, Miyazawa K, Rodgers A, Talati I, Lotan Y: Epidemiology of stone disease across the world. World J Urol. 2017, 35:1301-1320. 10.1007/s00345-017-2008-6

2. Ather MH, Talati J, Biyabani R: Physician responsibility for removal of implants: the case for a computerized program for tracking overdue double-J stents. Tech Urol. 2000, 6:189-192.

3. Sighinolfi MC, Micali S, De Stefani S, et al.: Indwelling ureteral stents and sexual health: a prospective, multivariate analysis. J Urol. 2007, 178:229-231. 10.1016/j.juro.2007.03.039

4. Al-Kandari AM, Al-Shaiji TF, Shaaban H, Ibrahim HM, Elshebiny YH, Shokeir AA: Effects of proximal and distal ends of double-J ureteral stent position on postprocedural symptoms and quality of life: a randomized clinical trial. J Endourol. 2007, 21:698-702. 10.1089/end.2007.9949

5. Nazim SM, Ather MH: Alpha-blockers impact stent-related symptoms: a randomized, double-blind, placebocontrolled trial. J Endourol. 2012, 26:1237-1241. 10.1089/end.2012.0036

6. Bhattar R, Tomar V, Yadav SS, Dhakad DS: Comparison of safety and efficacy of silodosin, solifenacin, tadalafil and their combinations in the treatment of double-J stent-related lower urinary system symptoms: a prospective randomized trial. Turk J Urol. 2018, 44:228-238. 10.5152/tud.2018.50328

7. Rosen R, Brown C, Heiman J, et al.: The Female Sexual Function Index (FSFI): a multidimensional selfreport instrument for the assessment of female sexual function. J Sex Marital Ther. 2000, 26:191-208. 10.1080/009262300278597

8. Rehman KU, Asif Mahmood M, Sheikh SS, Sultan T, Khan MA: The Female Sexual Function Index (FSFI): translation, validation, and cross-cultural adaptation of an Urdu version “FSFI-U.”. Sex Med. 2015, 3:244250. 10.1002/sm2.77

9. Akdeniz E, Bolat MS: Ureterorenoscopy with stenting and its effect on female sexual function . Urol J. 2017, 14:3059-3063.

10. Ather MH, Paryani J, Memon A, Sulaiman MN: A 10-year experience of managing ureteric calculi: changing trends towards endourological intervention--is there a role for open surgery?. BJU Int. 2001, 88:173-177. 10.1046/j.1464-410x.2001.02302.x

11. Ather MH, Nazim SM, Sulaiman MN: Efficacy of semirigid ureteroscopy with pneumatic lithotripsy for ureteral stone surface area of greater than $30 \mathrm{~mm}$. J Endourol. 2009, 23:619-622. 10.1089/end.2008.0182

12. Ather MH, Shrestha B, Mehmood A: Does ureteral stenting prior to shock wave lithotripsy influence the need for intervention in steinstrasse and related complications?. Urol Int. 2009, 83:222-225. $10.1159 / 000230028$

13. Joshi HB, Newns N, Stainthorpe A, MacDonagh RP, Keeley FX Jr, Timoney AG: Ureteral stent symptom questionnaire: development and validation of a multidimensional quality of life measure. J Urol. 2003, 169:1060-1064. 10.1097/01.ju.0000049198.53424.1d

14. Eryildirim B, Tuncer M, Kuyumcuoglu U, Faydaci G, Tarhan F, Ozgül A: Do ureteral catheterisation procedures affect sexual functions? A controlled prospective study. Andrologia. 2012, 44:419-423. 10.1111/j.1439-0272.2011.01199.x 


\section{Cureus}

15. Eryildirim B, Tuncer M, Sahin C, Yucetas U, Sarica K: Evaluation of sexual function in patients submitted to ureteroscopic procedures. Int Braz J Urol. 2015, 41:791-795. 10.1590/S1677-5538.IBJU.2014.0353

16. Fawzi R, Ali Z: Association of JJ stent insertion and sexual function: a cohort study [PREPRINT] . F1000Res. 2018, 10.12688/f1000research.16608.1

17. Bolat MS, Akdeniz E, Asci R, Erdemir F, Cinar O, Tomak L: Ureterorenoscopy with stenting and its effect on male sexual function: a controlled randomised prospective study. Andrologia. 2017, 49:9. 10.1111/and.12746 